\title{
Evolución del compromiso cardiovascular de pacientes insuficientes renales, en hemodiálisis, sin bloqueo del eje renina-angiotensina
}

\author{
Sonia Kunstmann ${ }^{1,2}$, Antonio Vukusich ${ }^{1,3}$, Luis Michea ${ }^{1}$, \\ Cristian Varela ${ }^{3}$, Irene Allende ${ }^{3}$, Sebastián Bravo ${ }^{1 a}$, \\ Daniela Gainzala, Daniela Sepúlvedala ${ }^{1 a}$ Elisa Marusic ${ }^{1}$, \\ Fernando Figueroa1.
}

\section{Cardiovascular assessment of non diabetic patients on hemodialysis}

Background: Diabetes mellitus is an important risk factor for cardiovascular complications among patients on hemodialysis. However, the incidence of these complications among non diabetic patients on hemodialysis is not well known. Aim: To assess the incidence of cardiovascular complications in non diabetic patients on hemodialysis. Patients and methods: Seventy five non diabetic patients aged 55.6 \pm 17 years ( 48 males), receiving bemodialysis three times a week were evaluated with laboratory tests, echocardiogram and a carotid ultrasound. In 26 patients, interleukin 6, tumor necrosis factor alpha, and intercellular adhesion molecule (ICAM-1) were also measured. Patients were followed during two years. Results: The mean lapse of dialysis therapy was $6.5 \pm 5$ years. The main cause of renal failure was bypertension. Sixty two percent had systolic bypertension, 86\% had concentric left ventricular bypertrophy, 43\% had atrial dilatation and 60\% had calcifications in the thoracic aorta. Compared with normal controls, patients had higher levels of interleukin 6, tumor necrosis factor alpha and ICAM-1. Carotid media thickness was also bigher and increased in the two years of follow up. No correlations were found between ventricular bypertrophy and dialysis lapse, packed red cell volume, calcium phosphorus product, parathormone levels or median arterial pressure. No cardiovascular events were recorded during the follow up period. Conclusions: Non diabetic patients on chronic bemodialysis have a high frequency of ventricular bypertrophy, carotid media thickening, aortic calcifications and an increase in proinflammatory cytokines (Rev Méd Chile 2009; 137: 351-60).

(Key words: Cytokines; Heart failure; Kidney failure, chronic)

\footnotetext{
Recibido el 10 de julio, 2008. Aceptado el 27 de noviembre, 2008.

Trabajo financiado por Proyectos Fondecyt № 1040338 (E.M.) y № 1020265 (F.F.)

${ }^{1}$ Facultad de Medicina, Universidad de los Andes. ${ }^{2}$ Clínica Las Condes. ${ }^{3}$ Clínica Dávila

anterno de medicina, Universidad de Los Andes. Santiago de Chile.
}

Correspondencia $a$ : Dra. Sonia Kunstmann F. Departamento Enfermedades Cardiovasculares, Clínica Las Condes. Lo Fontecilla 441, Las Condes, Santiago. Fax: 2430875.

E mail: skunstmann@clc.cl 
$\mathrm{E}^{\mathrm{n}}$ n Chile hay 812 pacientes con insuficiencia renal crónica (IRC) por millón/habitantes en hemodiálisis, la tasa más alta de América-Latina ${ }^{1}$, y en mayores de 45 años, se duplica cada ocho años. Pese a los avances en técnicas de hemodiálisis crónica (HD), se comunican resultados poco favorables para estos pacientes, con esperanza de vida de $1 / 5$ respecto de población general, por complicaciones y muerte cardiovascular ${ }^{2}$. A su vez, enfermedad coronaria, miocardiopatía hipertensiva, insuficiencia cardiaca y accidentes vasculares, son 5 veces más frecuentes en IRC que en población general. La hipertrofia ventricular izquierda (HVI) sería la alteración cardíaca más frecuente en hemodializados, representando un factor de riesgo independiente en estos pacientes $^{3}$. La diabetes mellitus, presente en aproximadamente 35\% de los pacientes en HD, es una de las principales causas de IRC, siendo un factor independiente de riesgo para desarrollar insuficiencia cardíaca, ateroesclerosis y daño vascular ${ }^{4}$.

Diferentes esfuerzos se realizan para disminuir el daño cardiovascular y evitar la aterosclerosis acelerada, de pacientes HD, mediante control exhaustivo de diabetes, hipertensión arterial (HTA), hipercolesterolemia o del metabolismo fosfo-cálcico. Sin embargo, la mayoría de los pacientes no logran reducir la HVI, la ateroesclerosis coronaria o mejorar la función ventricular y la mortalidad cardiovascular, primera causa de muerte $^{2}$.

No sabemos si esta alta prevalencia de morbimortalidad cardiovascular en IRC está en relación a diabetes (causa frecuente de IRC) o si la IRC juega un rol per se importante en el mal pronóstico cardiovascular de estos pacientes.

Estudios en animales de experimentación y en pacientes hipertensos o insuficientes cardiacos, han demostrado que antes de la aparición de lesiones ateromatosas, se detectan en mononucleares de sangre periférica elementos de defensa antioxidantes, moléculas de adhesión intercelular (ICAM-1), integrinas, e interleuquinas IL-1, entre otros. A su vez, IL-1 y el factor de necrosis tumoral (TNF) son inductores de quimioquinas como IL-8, que se consideran "proinflamatorias" por su efecto quimioatractante para neutrófilos 5,6 . Esta cascada de citoquinas podría ser responsable, en parte, de la vasculopatía de la IRC, por lo que aparece atractivo su estudio en pacientes con IRC.
Se conoce que el bloqueo del eje reninaangiotensina (RAS) ha demostrado ser beneficioso en retardar la aparición de complicaciones cardiovasculares y tendría efecto antiaterogénico, en pacientes de alto riesgo, especialmente hipertensos e insuficientes cardiacos 7,9 . Dado que pacientes con IRC tienen alto riesgo cardiovascular y que el tratamiento con bloqueadores del eje reninaangiotensina, por su efecto benéfico cardiovascular, podría impedirnos observar el daño cardiovascular provocado por la IRC, es que excluimos del estudio aquellos con inhibidores de enzima convertidora o antagonistas de angiotensina II.

El objetivo del estudio fue conocer las características cardiovasculares, de una población chilena con IRC terminal, de causa no diabética, en HD trisemanal, seguida por dos años.

\section{Material y MÉTOdo}

Se realizó estudio clínico, prospectivo, multicéntrico, de dos años de seguimiento, en 8 centros de diálisis de Santiago, en pacientes IRC, con más de tres meses de hemodiálisis trisemanal.

Se excluyeron pacientes con: insuficiencia hepática, diabetes mellitus, valvulopatías con indicación quirúrgica, cáncer y aquellos con inhibidores de enzima convertidora o antagonistas de angiotensina II.

Estudio clínico. Fueron caracterizados según antecedentes demográficos, historia, examen clínico, causa de IRC y antigüedad en HD. Se consignó terapia en uso, insuficiencia cardíaca según capacidad funcional (I-IV) de $\mathrm{NYHA}^{10}$. En todos se midió electrolitos plasmáticos, fosfatemia, calcemia, hormona paratiroidea (PTH), perfíl lipídico, hematocrito y eficacia de HD medida como porcentaje de reducción de urea (PRU). Según método estándar se midió presión arterial (PA), pre y posdiálisis ${ }^{11}$. Se consideró HTA un promedio de tres mediciones consecutivas de PA sistólica $\geq 140 \mathrm{mmHg}$ o diastólicas $\geq 90 \mathrm{mmHg}$, pre o poshemodiálisis.

Se realizó en forma centralizada, ciega, por un mismo operador, al inicio, al año y dos años de seguimiento, según metodología estándar: Rx tórax (dos proyecciones), ecocardiograma Doppler 
color bidimensional ATL HDI-3000 con transductor de 2,4 megaHertz ${ }^{12,14}$ y ecografía carotídea bilateral con transductor lineal alta resolución, en segmento distal de carótidas comunes, bifurcación y segmento proximal de carótidas internas. Se midió espesor de íntima-media de pared carotídea y presencia de placas ateromatosas ${ }^{15-17}$.

Se consideró HVI al aumento del índice de masa ventricular (IMV) o de grosores de septum y pared posterior del ventrículo izquierdo (VI).

Fue aprobado por Comités de Ética institucionales y áreas respectivas.

Se realizó seguimiento por dos años, registrándose hospitalizaciones, fallecimientos y sus causas.

Biología molecular, estudios serológicos e inmunotipificación. En 16 pacientes al azar con IRC, y 8 controles sanos, pareados por edad y sexo, se extrajo ARN en monocitos de sangre periférica separados mediante gradiente de Ficoll. Se determinó abundancia relativa de mARNs que codifican para activina A, e IL-18 por RT PCR cuantitativa. Las muestras fueron amplificadas en paralelo, utilizando partidores específicos para activina A, IL-18 y la subunidad 18 S ribosomal. Estos resultados se expresan como la razón de abundancia del transcrito de las citoquinas/transcrito $18 \mathrm{~S}$.

Los estudios serológicos y de inmunotipificación celular se efectuaron en 26 pacientes con IRC y 34 controles sanos, pareados por edad y sexo. El nivel sérico de IL-6, IL-17 y TNF $\alpha$, se cuantificó por Elisa (R\&D System). Activación y proliferación celular se cuantificó por incorporación de bromo- deoxiuridina $(\operatorname{BrdU})^{18}$ y la cuantificación de subpoblaciones linfocitarias se determinó por citometría de flujo ${ }^{19}$.

Estadística. Se utilizó prueba de t de Student para muestras no pareadas, análisis de varianza para comparación de variables continuas. Se realizó análisis de correlación lineal para investigar potencial relación entre variables de interés. Un valor de $\mathrm{P}<0,05$ fue considerado significativo.

\section{Resultados}

Población general. Incluimos 75 pacientes consecutivos, no diabéticos, sin bloqueo de RAS, en HD trisemanal. La edad promedio fue 55,6 \pm 17 años, 48 (64\%) hombres, 27 (36\%) mujeres. La antigüedad promedio en diálisis fue 6,9 $\pm 4,9$ años, con diagnóstico de IRC de 7,9 \pm 5,9 años de antigüedad. Durante el seguimiento, no hubo hospitalizaciones ni mortalidad cardiovascular.

De acuerdo al PRU, los pacientes recibían dosis de diálisis promedio de $69,4 \% \pm 8,9 \%$ los hombres y $73,2 \% \pm 10,1 \%$ las mujeres. No se encontraron diferencias en valores de laboratorio según sexo, ni tampoco a dos años de seguimiento (Tabla 1).

Se encontró alta prevalencia de alteraciones del metabolismo fosfo-cálcico, con hiperfosfatemia e hiperparatiroidismo secundario en 65,5\%; y aumento del producto Ca x P en $57,7 \%$ de los pacientes. Todos presentaron valores séricos elevados de PTH $(660,9 \pm$ $128 \mathrm{pg} / \mathrm{ml}), y$ de aldosterona $(24,6 \pm 22 \mathrm{ng} / \mathrm{dl})$.

Tabla 1. Parámetros de laboratorio en pacientes hemodializados según sexo

\begin{tabular}{|lccc|}
\hline $\begin{array}{l}\text { Características basales pacientes } \\
\text { en H D }\end{array}$ & $\begin{array}{c}\text { Resultado basal } \\
\mathbf{N}=\mathbf{7 5}\end{array}$ & Hombres & Mujeres \\
\hline Colesterol total (mg/dl) & $172 \pm 38$ & $164,6 \pm 36,1$ & $185,6 \pm 39,5$ \\
Col-HDL (mg/dl) & $38 \pm 10,6$ & $34,7 \pm 8$ & $43,8 \pm 12,3$ \\
Triglicéridos (mg/dl) & $207,8 \pm 136$ & $221 \pm 154$ & $183,6 \pm 93$ \\
Calcemia (mg/dl) & $9,2 \pm 0,9$ & $9,1 \pm 0,9$ & $9,2 \pm 0,9$ \\
Fosfatemia (mg/dl) & $5,7 \pm 1,7$ & $6,0 \pm 1,8$ & $5,2 \pm 1,4$ \\
Producto Ca x P & $52,4 \pm 16$ & $54,6 \pm 17,3$ & $47,8 \pm 13,7$ \\
Hematocrito (\%) & $30,4 \pm 0,7$ & $31,5 \pm 6,5$ & $28,3 \pm 4,8$ \\
\hline
\end{tabular}

Valor normal: calcemia 8,4-10,4 mg/dl; fósforo 2,7-4,5 mg/dl.

HD =Hemodiálisis crónica. 
Las causas de IRC fueron: HTA 48\%, desconocida 20\%, glomerulopatías 19\%, reflujo vesicoureteral $4 \%$ y misceláneas $9 \%$. Al inicio del estudio 2 pacientes tenían enfermedad coronaria y 8 tenían accidente cerebrovascular (10,6\%). El 44\% tenía tabaquismo activo, 62\% dislipidemia, 95\% sedentarismo y 59,5\% tenía HTA. Sólo 39,1\% recibía hipotensores. El 32\% usaba bloqueadores del calcio, 24\% aspirina ${ }^{\circledR}$ y $21 \%$ eritropoyetina. El $73 \%$ estaba en capacidad funcional I NYHA.

Evolución de presión arterial de pacientes IRC. La prevalencia de HTA prediálisis fue: 57\% para HTA sistólica y $26 \%$ para HTA diastólica, reduciéndose a $49 \%$ y $22 \%$, respectivamente posdiálisis, p <0,05. (Tabla 2).

La prevalencia de HTA sistólica y diastólica, pre y posdiálisis, fue mayor en hombres que en mujeres, $\mathrm{p}<0,05$ (Tabla 3). Esto no se modificó en seguimiento a dos años.

La Rx tórax inicial fue normal en 43\%, presentó congestión pulmonar en $4 \%$ de los pacientes (Tabla 4). A dos años de seguimiento, se encontró aumento significativo de calcificación aórtica y cardiomegalia, ( $\mathrm{p}<0,05$ para ambos parámetros).
Hipertrofia ventricular. El ecocardiograma mostró HVI concéntrica en $86 \%$ de los pacientes y dilatación auricular en $43 \%$. Sólo 12\% presentaba fracción de eyección anormal.

No se encontraron diferencias basales versus 24 meses de seguimiento, en crecimiento auricular, dilatación VI, ni en HVI (Tabla 4).

No encontramos correlación entre el índice de masa ventricular y la antigüedad de la IRC o el tiempo de permanencia en diálisis (Figura 1).

Tampoco se encontraron correlaciones significativas entre IMV y presión arterial media, presión sistólica, hematocrito, calcemia, fosfatemia, producto fosfo-cálcico o PTH (Figuras 2 y 3). Esto persistió, aun al separar la población según sexo.

Evolución en remodelación de arterias de conducción. El grosor media-íntima carotídeo encontrando fue mayor a lo publicado ${ }^{20}$ para población sana chilena ajustado por edad ( $\mathrm{p}$ $<0,01$ ), con grosor promedio basal de $0,83 \pm 0,05$ $\mathrm{mm}$. Este grosor aumentó significativamente a los 24 meses de seguimiento: promedio $0,89 \pm 0,07$ $\mathrm{mm}$, especialmente en carótidas internas (p $<0,01)$. No se encontró diferencias en número de

Tabla 2. Prevalencia H TA y cifras de presión arterial pre y postdiálisis

\begin{tabular}{|lcc|}
\hline & Prediálisis & Postdiálisis \\
\hline PA sistólica (mmHg) & $152,7 \pm 4,5$ & $138,8 \pm 4,5$ \\
Prevalencia HTA sistólica (\%) & 57 & 49 \\
PA diastólica (mmHg) & $84,4 \pm 4$ & $75,5 \pm 2,3$ \\
Prevalencia HTA diastólica (\%) & 36,4 & 17,5 \\
\hline
\end{tabular}

Valores de presión arterial de media semana. Promedio \pm DE

Tabla 3. Prevalencia de H TA pre y postdiálisis según sexo

\begin{tabular}{|lcccc|}
\hline & \multicolumn{2}{c}{ Hombres } & \multicolumn{2}{c|}{ Mujeres } \\
& Prediálisis & Postdiálisis & Prediálisis & Postdiálisis \\
\hline HTA sistólica (\%) & 61,7 & 20,5 & 30,8 & 19,2 \\
HTA diastólica (\%) & 29,8 & 21,3 & 11,5 & 7,7 \\
\hline
\end{tabular}

Valores de presión arterial de media semana. Promedio \pm DE 
Tabla 4. Ecocardiograma y radiografía inicial y a dos años de seguimiento

\begin{tabular}{|lcc|}
\hline & $\begin{array}{c}\text { Basal } \\
\text { Promedio } \pm \mathbf{D E}\end{array}$ & $\begin{array}{c}\text { 24 Meses } \\
\text { Promedio } \mathbf{D E}\end{array}$ \\
\hline Diámetro auricular Izquierdo (cm) & $36,1 \pm 0,9$ & $37,1 \pm 0,8$ \\
Grosor pared post VI (mm) & $12,5 \pm 0,39$ & $12 \pm 0,33$ \\
Grosor septum VI (mm) & $12,8 \pm 0,4$ & $12,3 \pm 0,38$ \\
Diámetro sistólico VI (mm) & $34,9 \pm 0,9$ & $36,6 \pm 1,8$ \\
Diámetro diastólico VI (mm) & $53,5 \pm 1,05$ & $51,4 \pm 1,5$ \\
Masa ventricular izquierda (gr) & $276,5 \pm 17$ & $269,6 \pm 17$ \\
Índice masa ventricular (gr/m $\left.{ }^{2}\right)$ & $162,6 \pm 9,3$ & $149,6 \pm 8,2$ \\
Calcificación aórtica en Rx tórax (\%) & 34 & $59,6^{*}$ \\
Cardiomegalia radiológica (\%) & 35 & $61,4^{*}$ \\
\hline
\end{tabular}

*p $=0,05$ para valor basal vs 24 meses. Valores normales: diámetro auricular izquierdo 1,9-4 cm; área $\leq 20$ $\mathrm{cm}^{2}$; IMV $\leq 134 \mathrm{gr} / \mathrm{m}^{2}$ en hombres; $\leq 110 \mathrm{gr} / \mathrm{m}^{2}$ en mujeres; grosores septum y pared posterior VI $\leq 11 \mathrm{~mm}$.

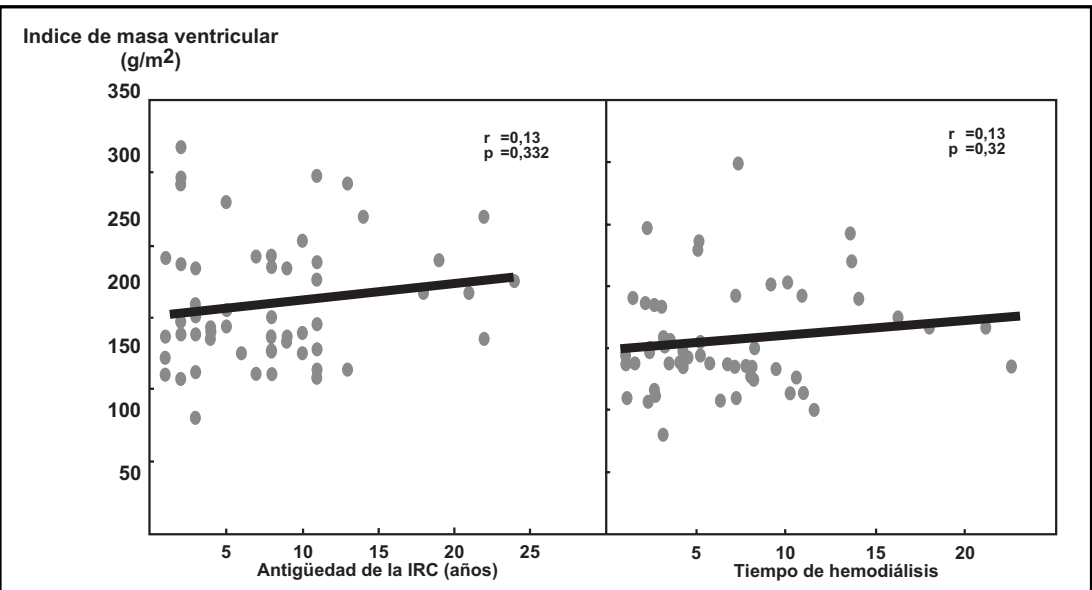

Figura 1. Correlación entre índice de masa ventricular (IMV), antigüedad de la IRC y del tiempo en tratamiento en HD. Análisis de regresión múltiple para evaluar impacto de la antigüedad de la IRC (izquierda) y el tiempo en HD (derecha) y la hipertrofia ventricular izquierda.

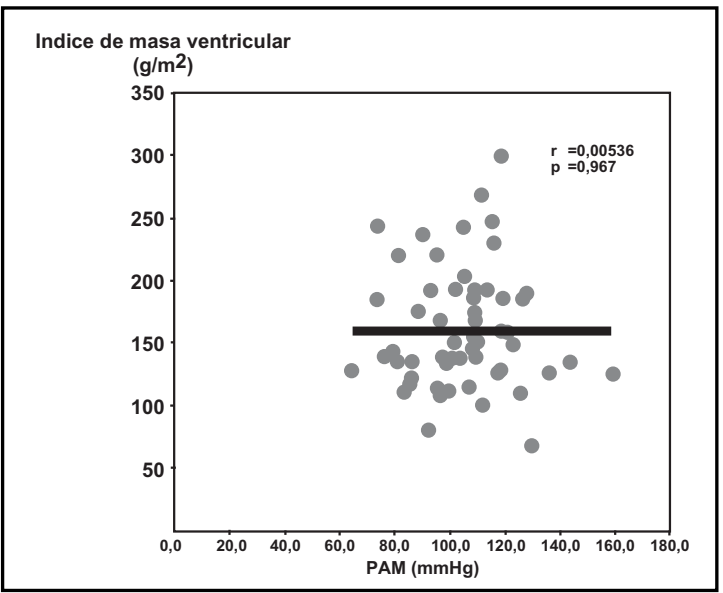

Figura 2. Correlación entre índice de masa ventricular y presión arterial media (PAM). Se realizó análisis de regresión múltiple. 


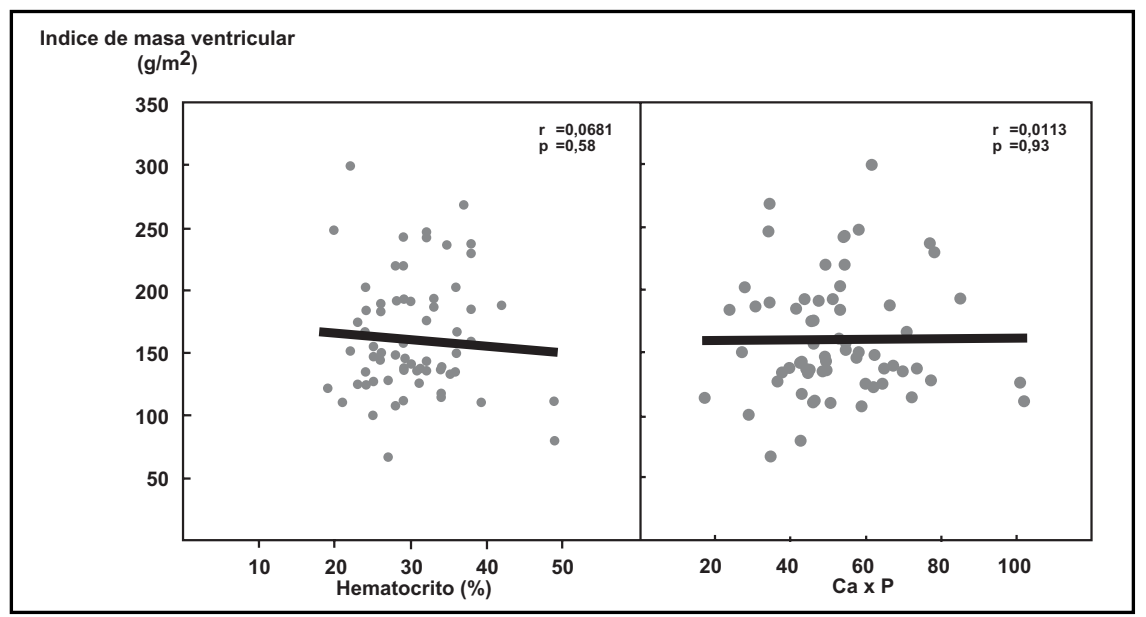

Figura 3. Correlación entre índice de masa ventricular, hematocrito y producto calcio-fósforo. Se realizó análisis de regresión múltiple para evidenciar posible relación entre hematocrito (izquierda) o producto CaxP e hipertrofia ventricular.

placas ateromatosas carotídeas basales versus dos años (Figura 4).

Citoquinas proinflamatorias y probipertróficas (Tabla 5). Comparado con controles sanos pareados por edad y sexo, encontramos incremento significativo en el nivel sérico de citoquinas proinflamatorias: IL-6 y TNF $\alpha$, en pacientes IRC, coincidente con descripciones previas ${ }^{5,21}$.
Pese al incremento de IL-6, no encontramos diferencias para IL-17, que se induciría por IL-6 6 .

El estudio de subpoblaciones linfocitarias demostró incremento significativo del porcentaje de linfocitos CD8+ con reducciones también significativas de linfocitos CD4+ y del índice CD4/CD8. Así mismo, encontramos aumento significativo de expresión de moléculas de adhesión intercelular ICAM-1 en linfocitos de sangre periférica. Pese a

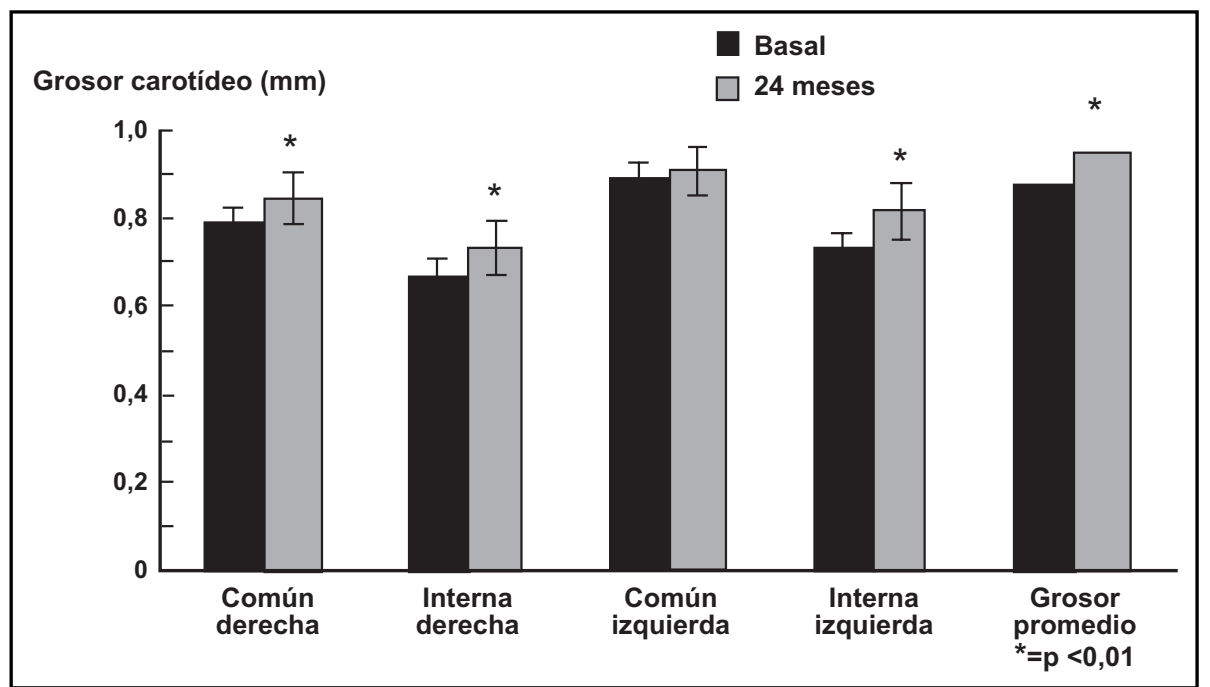

Figura 4. Grosor íntima-media carotídeo basal y a dos años. Las barras representan el valor promedio \pm DE. Asterisco indica $\mathrm{P}<0,01$. 
Tabla 5. Estudio inmunológico de pacientes con IRC

\begin{tabular}{|lccc|}
\hline $\begin{array}{l}\text { Citoquinas y subpoblaciones } \\
\text { linfocitarias }\end{array}$ & $\begin{array}{c}\text { Pacientes con IRC } \\
\text { en H D (n=26) }\end{array}$ & $\begin{array}{c}\text { Controles sanos } \\
(\mathbf{n = 3 4 )}\end{array}$ & Valor de $\mathbf{p}$ \\
\hline Interleuquina IL 6 & $11,2 \pm 9,8$ & $1,9 \pm 2,1$ & 0,002 \\
TNFa & $3,7 \pm 2,7$ & $0,07 \pm 0,2$ & 0.0001 \\
Linfocitos CD 8+ (\%) & $32 \pm 8,7$ & $26,7 \pm 9,1$ & 0,041 \\
Linfocitos CD 4+ (\%) & $43,3 \pm 8,7$ & $50,0 \pm 10,6$ & 0,03 \\
Indice CD4/CD8 & $1,4 \pm 0,5$ & $2,2 \pm 0,9$ & 0,004 \\
molécula de adhesión intercelular ICAM 1 & $22,8 \pm 10,3$ & $14,6 \pm 5,1$ & 0,041 \\
\hline
\end{tabular}

Promedio $\pm \mathrm{DE}$

que ello sugiere activación linfocitaria in-vivo, no detectamos diferencias entre pacientes y controles en los niveles de activación y proliferación celular in-vitro, como se ha descrito 22 .

También se encontró aumento significativo de citoquina inflamatoria IL-18 y de activina A, en pacientes con IRC, en relación a controles sanos, pareados por sexo y edad, $\mathrm{P}<0,05$ (Figura 5).

\section{DISCUSIÓN}

Se describen las características cardiovasculares y evolución a 2 años, de pacientes con IRC, de causa no diabética, sin bloqueo RAS. El haber excluido pacientes diabéticos del presente estudio, permite evaluar el grado de compromiso cardiovascular ateroesclerótico propio de la IRC, sin confundirlo con aquél provocado por diabetes ${ }^{23}$.

También excluimos pacientes IRC con bloqueo RAS, para evitar que el efecto cardiovascular potencialmente benéfico de esta terapia pudiera impedir observar el daño CV por la IRC. El bloqueo del eje renina-angiotensina ha demostrado retardar la aparición de complicaciones cardiovasculares, especialmente en hipertensos e insuficientes cardíacos de cualquier causa. Estas drogas mejorarían la HVI, la fibrosis miocárdica, el engrosamiento de la pared arterial, y tendrían efectos antiaterogénicos ${ }^{7-9}$.

La causa más frecuente de IRC en esta población seleccionada, fue HTA, observándose alta prevalencia de HTA sistólica (57\%), el doble que

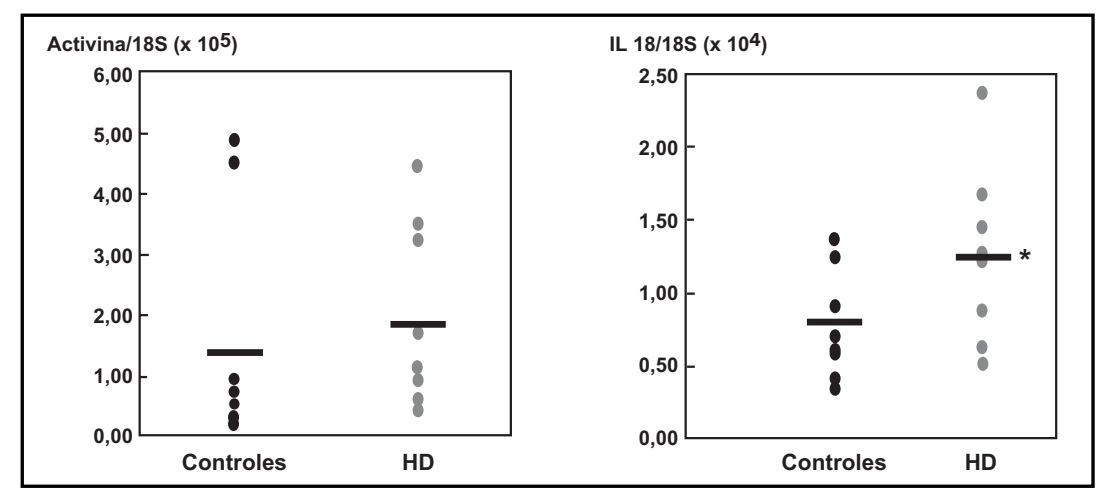

Figura 5. Niveles de activina A e IL-18 en hemodializados y controles. Los puntos representan el promedio de las determinaciones para cada muestra. La línea horizontal representa el promedio para cada grupo experimental. El asterisco indica $\mathrm{P}<0,05$. 
en población general. Sin embargo, fue menor que la reportada en poblaciones de IRC no seleccionadas, que incluyen pacientes diabéti$\cos ^{11,23}$.

Encontramos alta prevalencia de tabaquismo, sedentarismo y dislipidemia. La mayoría tenía colesterol total normal, HDL bajo y triglicéridos altos, lo que ha sido relacionado con inflamación crónica vascular, malnutrición y mayor mortalidad. Existen pocos estudios sobre dislipidemia que incluyan pacientes con IRC. Los estudios Heart Protection Study ${ }^{24}$ y CARE ${ }^{25}$, mostraron que reducir el colesterol era beneficioso independiente de la función renal.

$\mathrm{Al}$ inicio del estudio se encontró HVI concéntrica en $86 \%$ de los pacientes, porcentaje que no se modificó en dos años de seguimiento y que resultó ser independiente del tiempo transcurrido en hemodiálisis. Esto podría sugerir que la HVI se iniciaría precozmente en la IRC. Esta prevalencia de HVI es mayor a la descrita en poblaciones sin IRC. Wheeler et $\mathrm{al}^{26}$ comunicaron $41 \%$ de HVI concéntrica en pacientes que iniciaban $\mathrm{HD}$, con ecocardiograma normal en sólo 16\%. En nuestra población, aquellos sin HVI, tenían crecimiento auricular izquierdo, como manifestación precoz de cardiopatía, la mayoría con función ventricular sistólica normal.

De los factores contribuyentes a la alta prevalencia de HVI, podrían invocarse además de HTA, la anemia observada en IRC. Foley et $\mathrm{al}^{27}$ señalaron que por cada gramo de hemoglobina menor de $12 \mathrm{~g}$, el riesgo relativo de HVI es de 1,2; de insuficiencia cardiaca 1,28 y de mortalidad cardiovascular de 1,14. Sin embargo nuestro estudio no encontró correlación entre IMV y hematocrito.

Por otra parte, la HVI no correlacionó con la antigüedad de la IRC ni el tiempo de permanencia en HD, pero es posible que el diagnóstico de IRC fuera tardío. Tampoco encontramos correlación entre HVI y alteraciones del metabolismo fosfocálcico, o HTA.

De los factores contribuyentes a mayor rigidez arterial, causa invocada para $\mathrm{HVI}^{28-30}$, encontramos hipervolemia, HTA, anemia, microinflamación, activación del sistema renina-angiotensina-aldosterona $\mathrm{y}$ calcificaciones arteriales.

Las calcificaciones vasculares y las alteraciones del metabolismo fosfo-cálcico fueron frecuentes en el grupo estudiado. La prevalencia de calcificación de aorta torácica al término del estudio, fue cercana a 60\%. Blacher et al, estudiaron la relación entre rigidez arterial y calcificaciones de grandes vasos, demostrando que ambos son predictores de muerte cardiovascular ${ }^{31}$. Esto es relevante ya que calcificaciones vasculares, especialmente en aorta torácica, podrían predecir ateromatosis vascular en otros territorios de difícil exploración ${ }^{32,33}$.

El aumento del grosor de media-íntima carotídeo, es considerado un indicador de remodelación vascular y un buen predictor de eventos cardiovasculares $^{34,35}$. En esta población, el grosor promedio de media-íntima carotídeo fue mayor que el encontrado en poblaciones sanas chilenas ajustadas por edad $^{20}$. Por otra parte, el aumento significativo del grosor carotídeo, observado en dos años de seguimiento de esta población, sugiere progresión de la enfermedad ateroesclerótica.

En la IRC se describe ateroesclerosis acelerada con respuesta inflamatoria crónica de pared arterial, cuya primera manifestación aterogénica sería la disfunción endotelial que facilitaría la adherencia y migración de monocitos hacia la íntima. Estas células activadas, amplifican el proceso inflamatorio liberando citoquinas, que estimulan la adherencia y migración de linfocitos hacia la íntima y el reclutamiento de células musculares lisas. En este sentido, trabajos previos han reportado niveles plasmáticos de IL-18 elevados y peor pronóstico en pacientes con insuficiencia cardiaca post-isquémica y miocardiopatía dilatada ${ }^{36,37}$. En este estudio, encontramos aumento significativo de citoquinas proinflamatorias y prohipertróficas, entre ellas IL-6, IL-18 y TNF $\alpha$, como también de moléculas de adhesión intercelular ICAM 1 , en pacientes en HD. TNF $\alpha$ es potente inductor de moléculas de adhesión endotelial y su nivel sérico se correlaciona positivamente con proteína $\mathrm{C}$ reactiva y otros marcadores de daño vascular en IRC $^{5}$, y con mortalidad cardiovascular ${ }^{38}$. IL 18 es una citoquina prohipertrófica a nivel cardíaco $^{37}$, y activina A es una citoquina de la familia del TGF- $\beta$, que se eleva en pacientes insuficientes cardíacos y participa en la hipertrofia de cardiomiocitos en modelos experimentales 39,40 .

La importancia de IL-6 como citoquina proinflamatoria, ha sido objeto de marcado interés porque induce una nueva estirpe inflamatoria celular denominada Th-17, cuyo papel se desconoce en la IRC ${ }^{6}$.

Activina A también mostró tendencia al aumento, aunque no significativa. Trabajos futuros 
determinarán la importancia de estas citoquinas en pacientes HD y su correlación con la magnitud del daño cardiovascular en IRC.

Durante el seguimiento, no hubo hospitalizaciones ni mortalidad cardiovascular, lo que contrasta con lo reportado en poblaciones de IRC que incluyen diabéticos, en que la morbimortalidad cardiovascular es mayor. Un reciente estudio de Sanhueza et $\mathrm{al}^{23}$, de pacientes con IRC, reporta una mortalidad cardiovascular de $35 \%$ a 4 años. La evolución más favorable de nuestra población con IRC, posiblemente se explique por la exclusión de pacientes diabéticos.

La población estudiada mostró alta prevalencia de HVI, grosores altos de íntima-media carotí-

\section{REFERENCIAS}

1. Poblete H. XXIII Cuenta Anual de hemodiálisis crónica en Chile 2008. Sociedad Chilena de Nefrología.

2. Go A, Chertow G, Fan D, Mc Culloch, Hsu Ch. Chronic kidney disease and the risks of death, cardiovascular events, and hospitalization. $N$ Engl J Med 2004; 351: 1296-305.

3. Meeus F, Kourilsky O, Guerin A, Gaudry C, Marchais S, London G. Pathophysiology of cardiovascular disease in hemodialysis patients. Kidney Int 2000; 76: S140-7.

4. Lockhart C, Hamilton P, Mcveigh K, Mcveigh G. A cardiologist view of vascular disease in diabetes. Diabetes, Obesity \& Metabolism 2008; 10: 279-92.

5. Vaccaro F, Mule G, Cotttone S, Soresi M, GiannitrapaNI L. Circulating levels of adhesion molecules in chronic kidney disease correlate with the stage of renal disease and with C-reactive protein. Arch Med Res 2007; 38: 534-8.

6. Bettelli E, OukKa M, Kuchroo VK. T(H)-17 cells in the circle of immunity and autoimmunity. Nat Immunol 2007; 8: 345-50.

7. Amann K, Gassmann P, Buzello M, Reinhold O, Tornig $\mathrm{J}$. Effects of ACE inhibition and bradykinin antagonism on cardiovascular changes in uremic rats. Kidney International 2000, 58: 153-61.

8. Weber K. Aldosterone in Congestive Heart Failure. $N$ Engl J Med 2001; 345: 1689-97.

9. DZAU VJ. Mechanism of protective effects of ACE inhibition on coronary artery disease. Eur Heart $J$ 1998; 19: J2-J6.

10. The Criteria Committee of the New York Heart Association: Nomenclature and Criteria for Diagnosis. $9^{\text {th }}$ edition Boston. Little Brown, 1994.

11. Vukusich A, Fierro A, Morales J, Fantuzzi A, Vukusich C, Zehnder C. Epidemiología de la hiper- dea, que aumentaron a dos años de seguimiento, calcificaciones aórticas que también progresaron y elevados niveles de citoquinas inflamatorias, todo lo cual sugiere que la insuficiencia renal per se, es un factor de riesgo de enfermedad cardiovascular, que progresa, a pesar de diálisis trisemanal.

\section{Agradecimientos}

Los autores agradecen a médicos y enfermeras de los siguientes centros de diálisis, por su valiosa participación en este estudio: Diálisis Ltda., Hemodial, Nephrocare, Clínica Indisa, Instituto Nacional de Diálisis, Bilbao Dial, Padre Hurtado y Clínica Dávila, y a los pacientes, por su generosa colaboración.

tensión en hemodiálisis crónica. Rev Méd Chile 2002; 130: 610-15.

12. Chuang ML, Hibberd MG, Salton CJ. Importance of imaging method over imaging modality in noninvasive determination of left ventricular volume and ejection fraction. J Am Coll Cardiol 2000; 35: 477-84.

13. ACC/AHA/ASE Guidelines update for the clinical application of echocardiography. J Am Soc Echocardiography 2003; 16: 1091-110.

14. Devereux R, Reicher N. Echocardiographic determination of left ventricular mass in man: Anatomic validation of the method. Circulation 1977; 55: 613-8.

15. Kawagishi T, Nishizawa Y, Konishi T ET al. Highresolution B-mode ultrasonography in evaluation of atherosclerosis in uremia. Kidney Int 1995; 48: 820-6.

16. Burdick L, Pereti M, Salvaggio A. Relation between carotid artery atherosclerosis and time in dialysis. A non-invasive study in vivo. Clin Nephrol 1994; 42: 121-6.

17. Savage T, Clarke Al, Giles M, Tomson CRV, Raine AEG. Calcified plaque is common in the carotid and femoral arteries of dialysis patients without clinical vascular disease. Nephrol Dial Transplant 1998; 13 : 2002-12.

18. Holm M, Thomsen M, Høyer M , Hokland P. Optimization of a flow cytometric method for the simultaneous measurement of cell surface antigen, DNA content, and in vitro BrdUrd incorporation into normal and malignant hematopoietic cells. Cytometry 1998; 32: 28-36.

19. Carrion F, Fernández M, Iruretagiyena M, Coelho, Andrade L, Odete-Hilario M, Figueroa F. Selective depletion of $\mathrm{VB} 2+\mathrm{CD} 8+\mathrm{T}$ cells in peripheral blood from rheumatic heart disease patients. J Autoinmunity 2003; 20: 183-90.

20. Schargrodsky H, Hernández R, Champagne BM, Silva H, Vinueza R, Silva L et al. CARMELA: Assessment of 
Cardiovascular Risk in Seven Latin American Cities. Am J Medicine 2008; 121: 58-65.

21. Bolton CH, Downs LG, Victory JG. Endothelial dysfunction in chronic renal failure: roles of lipoprotein oxidation and proinflammatory cytokines. $\mathrm{Ne}$ phrol Dial Transplant 2001; 16: 1189-97.

22. Nairn J, Hodge G, Henning P. Changes in leukocyte subsets: clinical implications for children with chronic renal failure. Pediatr Nephrol 2005; 20: 190-6.

23. Sanhueza Me, Cotera A, Elgueta L, López G, Loncon P, MaCan F ET aL. Diabetes y hemodiálisis. Caracterización de una cohorte y seguimiento a cuatro años. Rev Méd Chile 2008; 136: 279-86.

24. Heart Protection Study Collaborative Group. Heart Protection Study of cholesterol lowering with simvastatin in 20.536 high-risk individuals: a randomized placebo-controlled trial. Lancet 2002; 360: 7-22.

25. Sacks F, Pfeffer M, Moye L, Brown L, Hamm P, Cole $T$ ET AL. Rationale and design of a secondary prevention trial of lowering normal plasma cholesterol levels after acute myocardial infarction: The Cholesterol and Recurrent Events Trial (CARE). Am J Cardiol 1991; 68: 1436-46.

26. Wheeler D, Townend J, Landray J. Cardiovascular risk factors in predialysis patients: Baseline data from the Chronic Renal Impairment in Birmingham study. Kidney Int 2003; 63: S201-03

27. Foley R, Parfrey P, Harnett J, Kent G, Murray D, BARRE P. The impact of anemia on cardiomyopathy, morbidity and mortality in end-stage renal disease. Am. J. Kidney Dis. 1996; 28: 53-56.

28. Gupta R, Birnbaum Y, Uretsky B. The renal patient with coronary artery disease: current concepts and dilemmas. J Am Coll Cardiol 2004; 44: 1343-53.

29. Covic A, Gusbeth-Tatomir P, Goldsmith D. Arterial stiffness in renal patients: An update. Am J Kidney Dis 2005; 45: 965-77.

30. London G, Marchais S, Guerin A, Metivier F, Adda H. Arterial structure and function in end-stage renal disease. Nephrol Dial Transplant 2002; 17: 1713-24.
31. Blacher J, Guerin A, Pannier B, Marchais S, London G. Arterial calcifications, arterial stiffness, and cardiovascular risk in end-stage renal disease. Hypertension 2001; 38: 938-42.

32. Guerin AP, London GM, Marchais SJ, Metivier F. Arterial Stiffness and vascular calcifications in endstage renal disease. Nephrol Dial Transplant 2001; 15: 1014-21.

33. Ishimura E, Okuno S, TANiwaki H. Different Risk Factors for Vascular Calcification in End-Stage Renal Disease between Diabetics and Nondiabetics: The Respective Importance of Glycemic and Phosphate Control. Kidney Blood Press Res 2008; 31: 10-5.

34. Touboul PJ, Hennerici MG, Meairs S. Mannheim intima-media thickness consensus. Cerebrovascular Dis 2004; 18: 346-9.

35. Bots ML. Carotid intima-media thickness as a surrogate marker for cardiovascular disease in intervention studies. Curr Med Res Opin 2006; 22: 2181-90.

36. Blankenberg S, Luc G, Ducimetiere P, Arveiler D, Ferrieres J, Amouyel P et al. Interleukin-18 and the risk of coronary heart disease in European men. Circulation 2003; 108: 2453-9.

37. Mallat Z, Heymes C, Corbaz A, logeart D, Alouani S, COHEN-Solal A ET AL. Evidence for altered interleukin (IL)-18 pathway in human heart failure. FASEB J 2004; 18: 1752-54

38. ANKer SD, Rauchhaus M. Insights into the pathogenesis of chronic heart failure: immune activation and cachexia. Curr Opin Cardiol 1999; 14: 211-6.

39. Mahmoudabady M, Mathieu M, Dewachter L, Hadad I, RAY L, JESPERS P ET AL. Activin-A, transforming growth factor-beta, and myostatin signalling pathway in experimental dilated cardiomyopathy. J Card Fail 2008; 14: 703-9.

40. Yndestad A, Ueland T, Oie E, Florholmen G, Halvorsen B, Attramadal H et al. Elevated levels of activin A in heart failure: potential role in myocardial remodeling. Circulation 2004; 109: 1379-85. 